Dr. Ross raises the very valuable point that patients with cardiac arrest who are far from emergency medical services may be at elevated risk, as compared with those living in a city. As such, the availability of an AED in the home might have particular value for a patient living in a rural area or in an area where emergency services are suboptimal. In our study, the number of events was too low to address this question in a definitive manner, but we can say that the rescue of patients with cardiac arrest by spouses certainly did occur in rural settings and far from medical aid. It is unlikely these patients would have survived ventricular fibrillation without an AED.
Gust H. Bardy, M.D.

Seattle Institute for Cardiac Research

Seattle, WA 98103-4819

gbardy@sicr.org

Kerry L. Lee, Ph.D.

Duke University

Durham, NC 27705

Jeanne E. Poole, M.D.

University of Washington

Seattle, WA 98195

1. Hohnloser SH, Kuck KH, Dorian P, et al. Prophylactic use of an implantable cardioverter-defibrillator after acute myocardial infarction. N Engl J Med 2004;351:2481-8.

\title{
HSV-2 Suppression and the Incidence of HIV
}

TO THE EDITOR: Watson-Jones et al. (April 10 issue $)^{1}$ report that the suppression of herpes simplex virus type 2 (HSV-2) does not decrease the incidence of human immunodeficiency virus (HIV) infection in female workers at recreational facilities in northwestern Tanzania. However, this finding is disputable, since treatment with $400 \mathrm{mg}$ of acyclovir twice daily did not suppress the incidence of HSV-2 infection. Any conclusion regarding the preventive effect of acyclovir should be based on the analysis of patients in whom the drug was detected, since the study is confounded because a majority of patients in the acyclovir group tested negative for acyclovir ( $>65 \%$ ) at both 12 months and 24 months. Similarly, the data on HIV acquisition should be evaluated according to the suppression of the HSV-2 viral load, as compared with baseline. We believe that the study's methodologic limitations prevent drawing any conclusions about the effect of acyclovir on HIV acquisition. $^{2}$

Andrea Lisco, M.D., Ph.D.

Christophe Vanpouille, Ph.D.

Eunice Kennedy Shriver National Institute of Child Health and Human Development

Bethesda, MD 20892

liscoa@mail.nih.gov

1. Watson-Jones D, Weiss HA, Rusizoka M, et al. Effect of herpes simplex suppression on incidence of HIV among women in Tanzania. N Engl J Med 2008;358:1560-71.

2. Cohen J. AIDS research: promising prevention interventions perform poorly in trials. Science 2007;317:440.

THE AUTHORS REPLY: We do not conclude that HSV-2 suppression is ineffective in the prevention of HIV acquisition but rather that suppressive therapy did not reduce the incidence of HIV acquisition in our study population. Despite intensive adherence support, study participants found it difficult to adhere to twice-daily use of acyclovir over a prolonged period. This finding is important, since it shows the limitations of this intervention as a practical measure in HIV control. Our results have been confirmed by a further trial showing no effect of acyclovir therapy on HIV incidence. ${ }^{1}$

Lisco and Vanpouille suggest that we should have restricted our analysis to patients in whom acyclovir was detected. Urine testing for acyclovir was done as a process measure in a small sample of subjects at selected time points. The presence of acyclovir at one time point is not a proxy for overall adherence during the trial. Similarly, HSV-2 shedding was measured only at certain visits, and there were too few subjects with shedding to conduct a subgroup analysis of HIV incidence. In any case, such analyses would have been subject to severe confounding, since an equivalent restriction would not have been possible in the placebo group, thus resulting in loss of the comparability provided by randomization.

Deborah Watson-Jones, M.D., Ph.D.

Helen A. Weiss, Ph.D.

Richard Hayes, D.Sc.

London School of Hygiene and Tropical Medicine

London WCIE 7HT, United Kingdom

deborah.watson-jones@lshtm.ac.uk

1. Celum C, Wald A, Hughes J, et al. Effect of acyclovir on HIV-1 acquisition in herpes simplex virus 2 seropositive women and men who have sex with men: a randomised, double-blind, placebocontrolled trial. Lancet 2008;371:2109-19. 